\title{
Bilateral superficial brachioulnar artery in a cadaver along with bilateral absence of palmaris longus
}

\author{
Sir, \\ We present a cadaveric case report which illustrates \\ superficial ulnar artery (SUA) bilaterally. This subject also \\ had a bilateral absence of palmaris longus and persistence \\ of median artery in the right limb, which makes this case \\ unique with a constellation of variations in both upper \\ limbs. We briefly review other reported cases to discuss \\ the occurrence of SUA variant and its importance.
}

The subject is a female cadaver of Indian ethnicity. No scar or incision marks were found on the upper limbs. On the right side, the ulnar artery arose as a branch from the brachial artery [Figure 1] and ran a superficial course just deep to the median cubital vein [Figure 2]. It then ran over the superficial forearm flexors and came to lie lateral to the flexor carpi ulnaris and ulnar nerve in the distal forearm [Figure 3]. The ulnar artery continued as the superficial palmar arch which anastomosed with the 'persistent' median artery which had coursed through the carpal tunnel along with the median nerve [Figure 4]. The median artery is an embryonic vessel that usually regresses during development. On the left side, the origin of the ulnar 
artery was similar to that of the right side [Figure 1] and ran near and deep to the basilic vein [Figure 2]. It coursed superficial to the superficial forearm flexors to reach the position in distal forearm lateral to flexor carpi ulnaris and ulnar nerve [Figure 3]. It continued as the superficial palmar arch which was completed by anastomosing with a palmar branch of the radial artery by burrowing the base of the thenar muscles [Figure 5]. Interestingly, palmaris longus muscle was absent in both the limbs [Figure 3].

The SUA is a potential hazard when trying to cannulate the superficial veins (median cubital vein or basilic vein) in the cubital fossa. There have been reports of inadvertent intra-arterial cannulation into the SUA in elbow ${ }^{[1]}$ on attempted 'intravenous' cannulation. Although rare, such accidents can result in disastrous outcome, if irritant

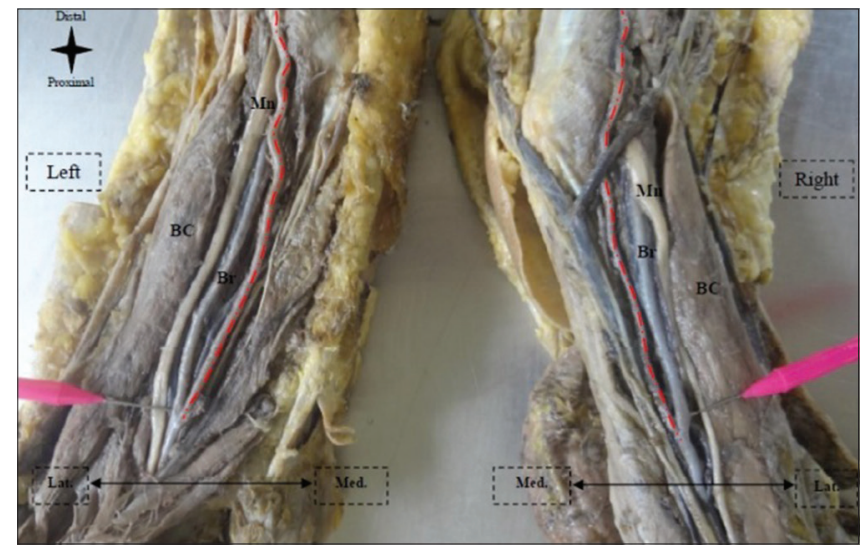

Figure 1: The photograph of both arms showing the origin of ulnar artery (pointed with pink pins) and its initial course (shown with red dashed lines). BC: Biceps brachii muscle, Mn: Median nerve, Br: Brachial artery, Med.: Medial, Lat.: Lateral

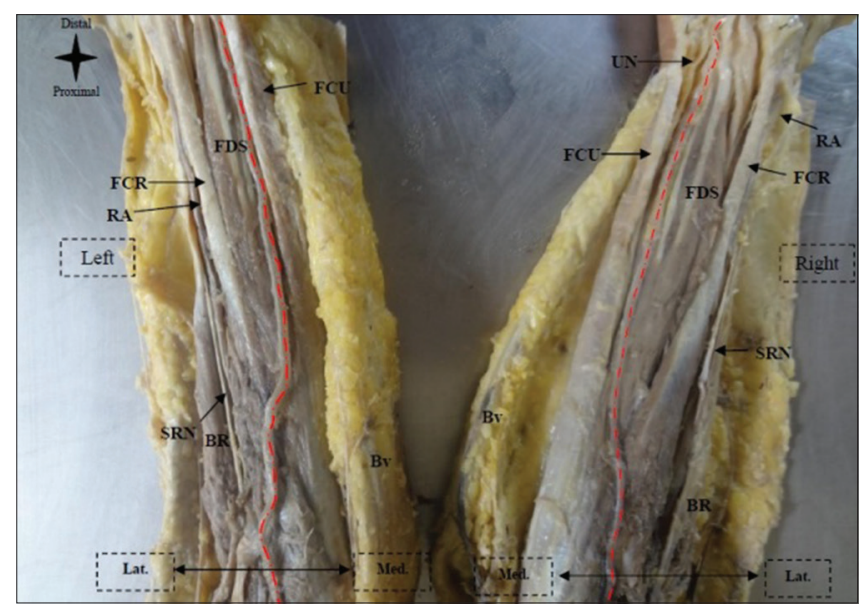

Figure 3: The photograph of both forearms showing the relation of ulnar artery (shown with red dashed lines) superficial to the forearm flexors to lie at the normal position distally. Note the absence of palmaris longus on both sides. Bv: Basilic vein, BR: Brachioradialis muscle, SRN: Superficial branch of radial nerve, FCR: Flexor carpi radialis, FDS: Flexor digitorum superficialis, FCU: Flexor carpi ulnaris, RA: Radial artery, UN: Ulnar nerve drugs are introduced, due to local ischaemia following vasospasm resulting in necrosis of tissue.

The SUA is also important in reconstructive surgeries. The first report of the superficial ulnar 'trap' was made by Fatah et al. as he first stated the importance of identifying this variant while raising a radial artery forearm flap..$^{[2]}$ The raising of a free forearm flap based on radial artery makes the upper limb solely dependent on the ulnar artery. However, if the ulnar artery is an SUA, then there is a risk of it being injured as it may be mistaken to be a superficial vein.

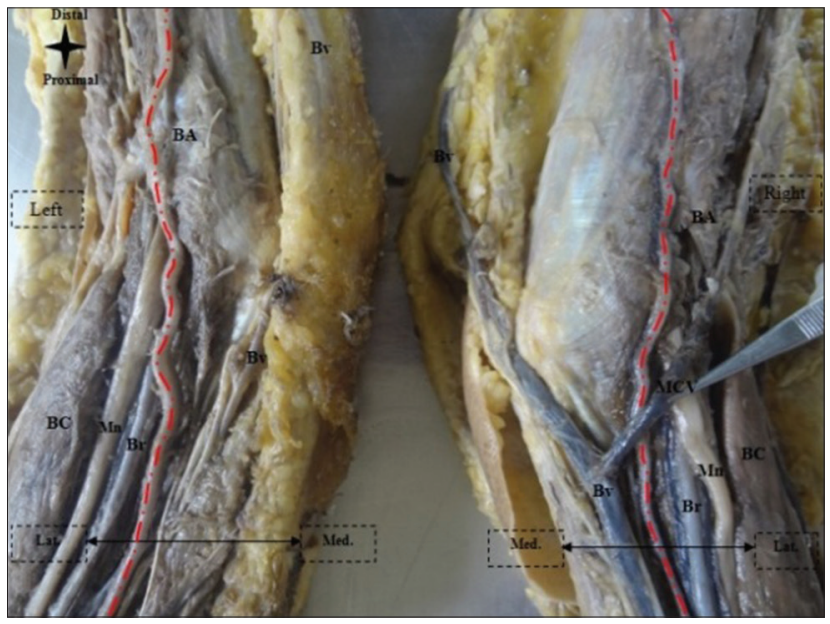

Figure 2: The photograph of both elbows showing the relation of ulnar artery (shown with red dashed lines) and the cubital fossa structures. Note the relation of the superficial veins to the artery. Note the relation of ulnar artery to the bicipital aponeurosis. BC: Biceps brachii muscle, Mn: Median nerve, Br: Brachial artery, Bv: Basilic vein, BA: Bicipital aponeurosis, MCV: Median cubital vein

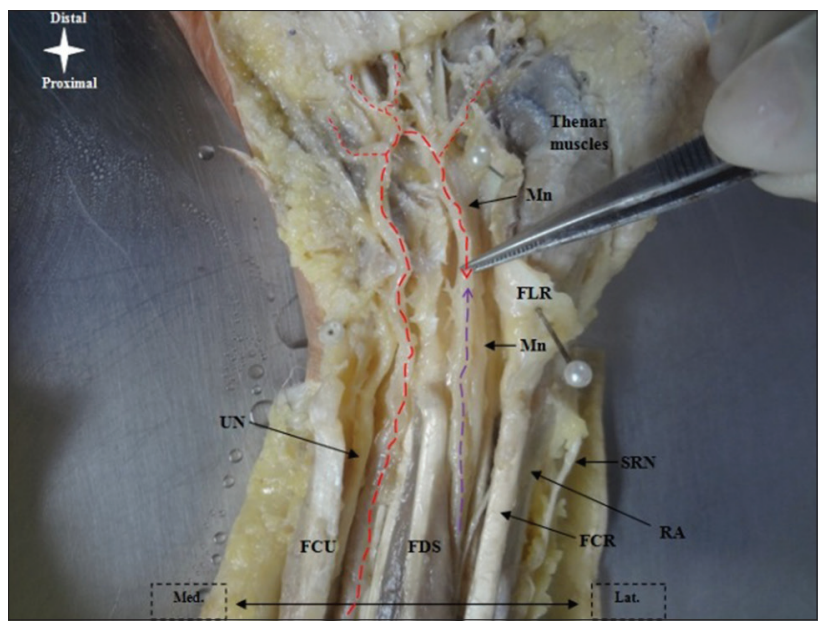

Figure 4: The photograph of the right hand showing the formation of superficial palmar arch (shown with red dashed lines) superficial to the flexor digitorum tendons. Palmar aponeurosis is reflected to the distal side. Note the anastomosis with the persistent median artery (shown with purple dashed lines). The flexor retinaculum is cut and reflected using stay pins. FCU: Flexor carpi ulnaris, FDS: Flexor digitorum superficialis, FCR: Flexor carpi radialis, RA: Radial artery, SRN: Superficial branch of radial nerve, Mn: Median nerve, FLR: Flexor retinaculum, UN: Ulnar nerve 


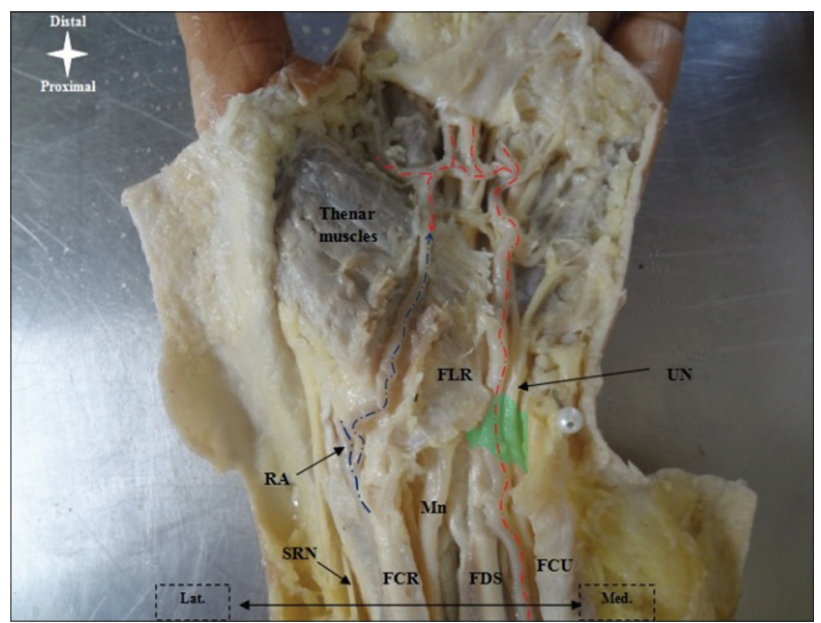

Figure 5: The left hand showing the formation of superficial palmar arch (shown with red dashed lines) superficial to the flexor tendons. Note the anastomosis with the superficial palmar branch of radial artery (shown with blue dashed lines) which is seen burrowing the thenar muscle fibres. The flexor retinaculum is intact. Guyon's canal is opened (shaded as green). FCU: Flexor carpi ulnaris, FDS: Flexor digitorum superficialis, FCR: Flexor carpi radialis, RA: Radial artery, SRN: Superficial branch of radial nerve, Mn: Median nerve, FLR: Flexor retinaculum, UN: Ulnar nerve

An interesting concurrence between the arterial variants and anomalies of palmaris longus has been observed in multiple reports. In one report by Yazar et al. ${ }^{[3]}$ there was inverse palmaris longus bilaterally along with SUA. In another report by Kachlik et al., ${ }^{[4]}$ there was a superficial brachiomedian artery with bitendinous palmaris longus.

In the study on 139 patients by Yadav et al., ${ }^{[5]}$ they intraoperatively recognised SUA in the two cases which had a unilateral absence of palmaris longus, but the other 137 cases including the six with bilateral absence of palmaris longus did not have SUA. They suggested that the unilateral absence of palmaris longus can be a warning sign for avoiding the superficial ulnar 'trap'..$^{[5]}$ It is surprising that Yadav et al. discovered an incredible 100\% sensitivity and $100 \%$ specificity for the SUA, if palmaris longus was absent unilaterally but not bilaterally. We understand that the conclusion by Yadav et al. may not be completely true in all cases as in our report, there is bilateral absence of palmaris longus with bilateral SUA, and there has also been a case where palmaris longus was seen in SUA variant, ${ }^{[6]}$ and as mentioned above, there were reported cases where aberrant morphologies of palmaris longus were seen with SUA variants. ${ }^{[3,4]}$

The present report and the brief review of previous reports show the importance of SUA in plastic surgery and in intravenous drug administration and an interesting pattern of palmaris longus anomalies/absence in some of the cases with SUA, while they were present in some.

\section{Acknowledgements}

The authors thank the staff of the dissection hall of the author's institution for their support.

\section{Financial support and sponsorship \\ Nil.}

\section{Conflicts of interest}

There are no conflicts of interest.

\section{Doris George Yohannan, Komalavallyamma Chandrakumari}

Department of Anatomy, Sree Gokulam Medical College and Research Foundation, Thiruvananthapuram, Kerala, India

\author{
Address for correspondence: \\ Dr. Doris George Yohannan, \\ Department of Anatomy, Sree Gokulam Medical College \\ and Research Foundation, Venjaramoodu, \\ Thiruvananthapuram, Kerala, India. \\ E-mail: dorisgeorge54@gmail.com
}

\section{REFERENCES}

1. Chin KJ, Singh K. The superficial ulnar artery - A potential hazard in patients with difficult venous access. $\mathrm{Br} \mathrm{J}$ Anaesth 2005;94:692-3.

2. Fatah MF, Nancarrow JD, Murray DS. Raising the radial artery forearm flap: The superficial ulnar artery "trap". Br J Plast Surg 1985;38:394-5.

3. Yazar F, Kirici Y, Ozan H, Aldur MM. An unusual variation of the superficial ulnar artery. Surg Radiol Anat 1999;21:155-7.

4. Kachlik D, Hajek P, Konarik M, Krchov M, Baca V. Coincidence of superficial brachiomedian artery and bitendinous palmaris longus: A case report. Surg Radiol Anat 2016;38:147-51.

5. Yadav PS, Ahmad QG, Shankhdhar VK, Nambi GI. Absence of the palmaris longus is a warning sign for avoiding the superficial ulnar artery 'trap'. Indian J Plast Surg 2013;46:149-50.

6. Casal D, Pais D, Toscano T, Bilhim T, Rodrigues L, Figueiredo I, et al. A rare variant of the ulnar artery with important clinical implications: A case report. BMC Res Notes 2012;5:660.

This is an open access article distributed under the terms of the Creative Commons Attribution-NonCommercial-ShareAlike 3.0 License, which allows others to remix, tweak, and build upon the work non-commercially, as long as the author is credited and the new creations are licensed under the identical terms.

\begin{tabular}{|l|l|}
\hline \multicolumn{2}{|c|}{ Access this article online } \\
\hline Quick Response Code: & Website: \\
\hline
\end{tabular}

How to cite this article: Yohannan DG, Chandrakumari K. Bilateral superficial brachioulnar artery in a cadaver along with bilateral absence of palmaris longus. Indian J Plast Surg 2017;50:108-10.

(C) 2017 Indian Journal of Plastic Surgery | Published by Wolters Kluwer - Medknow 